\title{
Surgical Outcomes of Congenital Heart Disease in Down Syndrome: Tertiary Centre Experience - Focus on the Electrical Conduction System
}

\author{
Kuwait University \\ Joud Al Balool \\ Kuwait University \\ Asia Al Saegh \\ Kuwait University \\ Hesham Menshawi \\ Ministry of Health \\ Nasreldeen Almeeri \\ Ministry of Health \\ Moustafa Elsayed \\ Ministry of Health \\ Faisal Al-Saiedi \\ Ministry of Health \\ Vadim Lyubomudrov \\ Ministry of Health
}

Mohammad Ebrahim ( $\nabla$ mohammad.ebrahim@ku.edu.kw)

\section{Article}

Keywords: Down syndrome, pacemaker, conduction disorder, surgical outcomes

Posted Date: March 1st, 2022

DOI: https://doi.org/10.21203/rs.3.rs-1389228/v1

License: (a) This work is licensed under a Creative Commons Attribution 4.0 International License. Read Full License 


\section{Abstract}

Purpose: To document outcomes of cardiac surgical repair in Down syndrome (DS) patients with specific focus on the associated electrical conduction morbidities, ultimately leading to a higher incidence of pacemaker implantation (PMI).

Methods: A retrospective study conducted between 2011 to 2020 .

Results: A total of 167 DS patients undergoing 204 surgeries were included. The mean gestational age (GA) and mean weight were 37.3 weeks and $5.5 \mathrm{~kg}$ respectively. Complete atrioventricular septal defect (AVSD) was the most common diagnosis. Pre-operative ECG revealed superior axis deviation (SAD) in $92 \%$ and $32 \%$ of patients with AVSD and isolated perimembranous ventricular septal defect (VSD), respectively $(p<0.01)$. Postoperative right bundle branch block (RBBB) was observed in $83 \%$ of patients post AVSD repair, and in $55 \%$ of patients following perimembranous VSD repair $(p=0.04)$. Ten patients underwent post-operative pacemaker implantation (PMI). Reintervention rate was around $8.9 \%$. Three mortalities were encountered throughout the study period, 2 of which were in-hospital deaths.

Conclusion: Low mortality was observed, however, a higher rate of PMI requirements noted with risk factors including lower age and weight.

\section{Introduction}

Approximately one-half of Down syndrome (DS) patients are born with congenital heart disease (CHD), with atrioventricular septal defect (AVSD) being the most common underlying lesion [1-4]. Other frequent lesions include atrial (ASD) and ventricular septal defects (VSD), along with tetralogy of Fallot (TOF) [1]. While surgical morbidities in DS patients are high $[5,6]$, reported mortality rates has been conflicting $[1-4$, 7]. As such, we thought to clarify the mortality data previously reported and focus specifically on the electrical conduction morbidities associated with DS patients, ultimately predisposing such group to a higher incidence of post-operative persistent heart block requiring pacemaker implantation (PMI).

In Kuwait, CHD screening is performed to all DS patients after birth through early pediatric cardiology consultation with electrocardiography (ECG) and echocardiography, mainly at Chest Diseases Hospital $(\mathrm{CDH})$. The $\mathrm{CDH}$ is the only tertiary center that conducts pediatric cardiac surgery in Kuwait. There are currently limited publications regarding the main objectives of this study.

\section{Methods}

\subsection{Study population}

This is a retrospective review conducted at CDH from January 2011 to May 2020. DS patients with history of cardiac surgery were identified via a surgical database created in 2008 , which includes all 
pediatric cardiac surgeries performed at $\mathrm{CDH}$. The ethics committee at both the Kuwait University Faculty of Medicine and the Ministry of Health approved the study protocol.

\subsection{Variables}

A review of the details regarding patient's baseline characteristics, operative techniques and postoperative data were conducted. Pre-operative characteristics including gender, anthropometric measures, baseline ECG parameters, and associated medical comorbidities were captured. Intra-operative data involved surgical descriptions of the operative techniques, cardiopulmonary bypass (CPBT) and aortic cross-clamping times (ACCT). Post-operative data analysis consisted of the length of hospital and intensive care unit (ICU) stay, duration of mechanical ventilatory support, in-hospital mortality, and other surgical complications including the need for PMI.

\subsection{Definitions and outcomes}

Hospital death was defined as death occurring during the post-operative hospital stay. The need for mechanical ventilatory support for more than 2 days was defined as prolonged intubation.

Main outcome data assessed in this study included post-operative ECG abnormalities and persistent heart block requiring PMI. Furthermore, other outcome data included post-operative complications such as prolonged post-operative mechanical ventilation, open-chest, bleeding, infections, effusions, pneumothorax, neurological insults (including vocal cord and phrenic nerve injuries), in addition to patient demise.

\subsection{Primary surgical techniques}

Two-patch technique (TPT) was utilized for complete AVSD lesions with a large VSD component [8], versus single-patch technique (SPT) was performed for AVSD with a small VSD component, as described previously [9]. Otherwise, Gore-Tex patch was typically used for the closure of a large isolated perimembranous VSDs and autologous pericardium was used for the closure of an isolated large ASDs. Furthermore, extended end-end anastomosis was implemented for all coarctation repairs in this cohort. Moreover, transannular patch, or valve-sparing technique if feasible, was performed for associated TOF lesions.

\subsection{Statistical analysis}

Statistical analysis was performed with the R software (version 4.0.5; R Foundation for Statistical Computing, Vienna, Austria). Frequencies were used to summarize categorical variables, whereas continuous variables were expressed as mean \pm standard deviation or median \pm range, as appropriate. Differences in categorical variables were compared using chi-square and variations in means were compared using t test. $P$ values were considered significant when $<0.05$.

\section{Results}




\subsection{Patients characteristics}

A total of 167 DS patients undergoing 204 CHD surgical repairs were included, comprising around $13 \%$ of all intra-cardiac surgeries performed at $\mathrm{CDH}$ (during the study period). The mean follow-up time was 48.1 months $( \pm 35.3)$. Eighteen patients were lost to follow-up. Main pre-, intra-, and post-operative parameters are summarized in Table 1. The mean gestational age (GA) was 37.3 weeks and the mean weight at initial surgery was $5.5 \mathrm{~kg}$ for the entire cohort. Major pre-operative comorbidities are shown in Table 2, with pre-operative hypothyroidism being the most common association. Pre-operative ECG characteristics are mentioned in Table 3. Superior axis deviation (SAD) was observed in $92 \%$ of patients with AVSD, compared to only $37 \%$ in patients with isolated perimembranous VSD $(p<0.01)$.

\subsection{Operative characteristics}

Primary cardiac pathology and types of surgeries are described in Table 4. Complete AVSD was the most associated CHD in patients with DS and repaired mostly using TPT. Single-ventricle physiology (unbalanced AVSD) patients only represented $2.4 \%$ of the cohort with one patient ultimately palliated with the Fontan operation.

\subsection{Postoperative complications}

Post-operative RBBB was observed in $83 \%$ of patients post AVSD repair, compared to $55 \%$ of patients following perimembranous VSD repair, $p=0.04$ (Table 3 ). Otherwise, prolonged intubation was the most observed postoperative complication (Table 5). The median stay for both ICU and total hospital duration were 5 (range: 1-67) and 15.5 (range: 5-121) days, respectively. Ten patients had persistent postoperative high-grade atrioventricular block (AVB) requiring PMI (Table 6; 5 with complete AVSD, 4 with perimembranous VSD and 1 with AVSD/TOF), with lower age and weight being statistically significant parameters. Redo surgery rate excluding PMI was at $8.9 \%$, with 6 patients undergoing catheter interventions as well (Tables 7 and 8). Three mortalities were encountered throughout the study period, 2 of which were in-hospital deaths (due to sepsis and acute shunt thrombosis).

\section{Discussion}

Recently, the rate for post-operative PMI for all intra-cardiac surgeries performed at CDH had been published [10]. It was noteworthy that the proportion of patients with DS in the pacemaker group was significantly higher [10]. Therefore, we thought to look at all DS patients' surgical outcomes and focus on the main pre- and post-operative electrical conduction disturbances.

Previously, DS had been shown to be a significant risk factor for developing AVB following cardiac repair $[1,11]$. During the same study period, the incidence of PMI in this DS cohort is high at $6 \%$, versus the overall $1.8 \%$ incidence for all patients undergoing intra-cardiac repair at $\mathrm{CDH}$ [10]. While smaller age, less weight and longer surgical times are potentially important risk factors accounting for this higher incidence (Table 6) [10], the associated electroanatomic variations commonly seen in DS patients are likely to be also contributing. 
First of all, the position of the papillary muscles are associated with the location of the conduction system, and are typically counterclockwise rotated secondary to the alterations of the heart tube in AVSD lesions [12]. Furthermore, the SAD mostly seen in DS with AVSD lesions is thought to be partly related to the posterior displacement of the atrioventricular node (AVN), along with the associated hypoplastic left anterior fascicle and the early onset of the left posterior fascicle branch $[13,14]$. Perhaps the mere inferior displacement of the AVN is the main culprit for the higher risk of post-operative heart block, rather than DS itself as its been previously shown that the incidence of PMI in non-syndromic AVSD patients is rather similar [10].

While it is known that SAD is associated commonly with DS septal defects, the exact incidence has not been previously well documented in the literature. Herein, $92 \%$ of AVSD patients had SAD, compared to only $35 \%$ in patients with perimembranous VSD. Possibly suggesting further displacement of the AVN, due to the additional primum septal deficiency, among the AVSD group $[15,16]$. Therefore, the association between SAD and perimembranous VSDs is less common [13].

To the best of the authors' knowledge, the incidence of postoperative RBBB in DS undergoing AVSD or perimembranous VSD has not been previously published. The incidence in this cohort was documented as $82 \%$ and $55 \%$, respectively. Such rates are similar to those seen following TOF repair [17]. This high incidence is due to the inadvertent injury of the proximal right bundle due to its proximity to the VSD infero-posterior margin, with suture impingement causing infarction of proximal right bundle [18].

Finally, the reintervention rates have been reasonable throughout the study period $[19,20]$, with extremely low mortality rates that is lower than most reported literature $[2-4,6,7,19-21]$. It's been observed that the frequency of more complex left atrioventricular valve anomalies is higher in non-syndromic AVSD lesions, perhaps contributing to the better surgical outcomes in DS patients $[3,19]$.

The usual limitations of a single center retrospective study, such as selection bias and inter-observer variability for clinical and echocardiographic evaluations performed by different physicians, apply to this data analysis. The small number of subjects limits statistical power and the feasibility of subgroup analysis in the cohort of the current study. Furthermore, the database used does not capture patients with DS who died prior to any cardiac surgery.

In conclusion, DS cardiac repair outcomes were excellent with low mortality, and with infrequent reintervention rates. A higher PMI rate was noted and was associated with lower age and weight at surgery. Electrical conduction disturbances commonly seen with DS patients likely contributed to the high PMI rate.

\section{Declarations}

Acknowledgements: 
We thank Omar Ibrahim Ibrahim (premedical student) for his contribution to the data collection for this project.

Authors contribution - 1) Mohammad Ebrahim - Conceptualization, methodology, ethics paperwork, article drafting, revision, concept, and design ;2) Joud Al Balool - Ethics paperwork, literature search, article drafting, and data collection ; 3) Asia Al Saegh - Ethics paperwork, article drafting, and data collection ; 4) Hisham H. Menshawi - Critical revision of article ; 5) Moustafa Elsayed - Critical revision of article ; 6) Critical revision of article ; 7) Faisal M. Alsaidi - Critical revision of article ; 8) Vadim Lyubomudrov - Critical revision of article, database organizer.

\section{Compliance with Ethical Statements}

Conflict of Interest: The authors declare that they have no conflict of interest.

Funding: There is no funding source.

Ethical approval: This article does not contain any studies with human participants or animals performed by any of the authors.

Availability of data and material: Available

\section{References}

1. Fudge Jr, J.C., et al., Congenital heart surgery outcomes in Down syndrome: analysis of a national clinical database. Pediatrics, 2010. 126(2): p. 315-322.

2. Anaclerio, S., et al., Conotruncal heart defects: impact of genetic syndromes on immediate operative mortality. Italian Heart Journal, 2004. 5: p. 624-628.

3. Formigari, R., et al., Better surgical prognosis for patients with complete atrioventricular septal defect and Down's syndrome. The Annals of thoracic surgery, 2004. 78(2): p. 666-672.

4. Santos, F.C.G.B., et al., Surgical treatment for congenital heart defects in Down syndrome patients. Brazilian journal of cardiovascular surgery, 2019. 34: p. 1-7.

5. Lal, P.S., et al., Surgical outcome of congenital heart disease in Down's syndrome. Asian Cardiovascular and Thoracic Annals, 2013. 21(2): p. 166-169.

6. Malec, E., et al., Results of surgical treatment of congenital heart defects in children with Down's syndrome. Pediatric cardiology, 1999. 20(5): p. 351-354.

7. Reller, M.D. and C.D. Morris, Is Down syndrome a risk for poor outcome in after repair of congenital heart defects? The Journal of pediatrics, 1998. 132(4): p. 738-741.

8. Daebritz, S.H., Correction of complete atrioventricular septal defects with two patch technique. Operative Techniques in Thoracic and Cardiovascular Surgery, 2004. 9(3): p. 208-220.

9. Nicholson, I.A., et al., Simplified single patch technique for the repair of atrioventricular septal defect. The Journal of thoracic and cardiovascular surgery, 1999. 118(4): p. 642-647. 
10. Ebrahim, M.A., et al., Pacemaker implantation post congenital heart disease surgical repair: tertiary center experience. European Journal of Pediatrics, 2020. 179(12): p. 1867-1872.

11. Tucker, E.M., et al., Permanent pacemaker for atrioventricular conduction block after operative repair of perimembranous ventricular septal defect. Journal of the American college of cardiology, 2007. 50(12): p. 1196-1200.

12. Hakacova, N., G.S. Wagner, and S.F. Idriss, Electroanatomic relationships in patients with primum atrioventricular septal defect. JACC: Cardiovascular Imaging, 2009. 2(12): p. 1357-1365.

13. Caro, M., et al., The electrocardiogram in Down syndrome. Cardiology in the Young, 2015. 25(1): p. 814.

14. Waldmann, V., et al., Atrial flutter catheter ablation in adult congenital heart diseases. Indian Pacing and Electrophysiology Journal, 2021. 21(5): p. 291-302.

15. Thiene, G., et al., Surgical anatomy and pathology of the conduction tissues in atrioventricular defects. The Journal of thoracic and cardiovascular surgery, 1981. 82(6): p. 928-937.

16. Suzuki, K., et al., Atypical form of atrioventricular septal defect without left axis deviation: relation between morphology and unusual QRS axis. Heart, 1993. 70(2): p. 180-184.

17. Hazan, E., et al., Is right bundle branch block aviodable in surgical correction of tetralogy of Fallot? Circulation, 1980. 62(4): p. 852-854.

18. Horowitz, L.N., J.A. Alexander, and L.H. Edmunds Jr, Postoperative right bundle branch block: identification of three levels of block. Circulation, 1980. 62(2): p. 319-328.

19. Lange, R., et al., The presence of Down syndrome is not a risk factor in complete atrioventricular septal defect repair. The Journal of thoracic and cardiovascular surgery, 2007. 134(2): p. 304-310.

20. Dodge-Khatami, A., et al., Outcomes and reoperations after total correction of complete atrioventricular septal defect. European journal of cardio-thoracic surgery, 2008. 34(4): p. 745-750.

21. Al-Hay, A.A., et al., Complete atrioventricular septal defect, Down syndrome, and surgical outcome: risk factors. The Annals of thoracic surgery, 2003. 75(2): p. 412-421.

\section{Tables}


Table 1

Main pre-, intra- and post-operative parameters.

Pre, intra and postoperative parameters

Pre-operative:

- Male, $n^{1}(\%)$

- GA, wks² (mean)

- Age at initial surgery, $\mathrm{mo}^{3}$ (mean)

- Weight at initial surgery, $\mathrm{kg}^{4}$ (mean) Intra-operative:

- $\mathrm{CPBT}^{5}$, mins $^{6}$ (mean)

- $\mathrm{ACCT}^{7}$, mins (mean)

Post-operative:

- Hospital stay, $d^{8}$ (median)

- ICU stay, d (median)

- Mortality

${ }^{1}$ Number

${ }^{2}$ Weeks

${ }^{3}$ Months

${ }^{4}$ Kilograms

${ }^{5}$ Cardiopulmonary Bypass Time

${ }^{6}$ Minutes

${ }^{7}$ Aortic Cross-Clamp Time

${ }^{8}$ Days
81 (48\%)

$37.3 \pm 1.5$

$8.8( \pm 16.1)$

$5.5( \pm 3.6)$

$71.9( \pm 30.7)$

$52( \pm 28.1)$

$15.5(5-121)$

$5(1-67)$

3 (1 late) 
Table 2

Major pre-operative comorbidities.

\begin{tabular}{|ll|}
\hline Major pre-operative comorbidities & $\mathbf{N}^{1}(\%)$ \\
\hline Hypothyroidism & $32(19 \%)$ \\
\hline $\mathrm{GI}^{2}$ disorders: & $4(2.4 \%)$ \\
- Duodenal atresia & $1(0.6 \%)$ \\
- NEC & $1(0.6 \%)$ \\
- Imperforate anus & $1(0.6 \%)$ \\
- Hirschsprung disease & \\
\hline Renal disease & $2(1.2 \%)$ \\
\hline${ }^{1}$ Number \\
\hline 2 \\
\hline 2 Gastrointestinal \\
\hline${ }^{3}$ Necrotizing Enterocolitis \\
\hline
\end{tabular}

Table 3

Pre, and post-operative electrocardiographic findings.

\begin{tabular}{|c|c|c|c|}
\hline Diagnosis & AVSD $^{1}$ & PMVSD $^{2}$ & P-value \\
\hline Pre-op ${ }^{3} S A D^{4}$ & $92 \%$ & $37 \%$ & $<0.01$ \\
\hline Post-op ${ }^{5}$ RBBB $^{6}$ & $83 \%$ & $55 \%$ & 0.04 \\
\hline \multicolumn{4}{|c|}{${ }^{1}$ Atrioventricular Septal Defect } \\
\hline \multicolumn{4}{|c|}{${ }^{2}$ Perimembranous Ventricular Septal Defect } \\
\hline \multicolumn{4}{|l|}{3 Pre-operative } \\
\hline \multicolumn{4}{|c|}{${ }^{4}$ Superior Axis Deviation } \\
\hline \multicolumn{4}{|l|}{5 Post-operative } \\
\hline${ }^{6}$ Right Bundle B & nch Bloc & & \\
\hline
\end{tabular}


Surgical procedures for the entire cohort.

\begin{tabular}{|c|c|}
\hline Surgical procedures & $\mathbf{N}^{1}(\%)$ \\
\hline Complete AVSD ${ }^{2}$ : & $71(42 \%)$ \\
\hline - Two-patch technique: & 54 \\
\hline o Prior $\mathrm{PAB}^{3}$ & 4 \\
\hline$o+\mathrm{Co}^{4}$ repair & 2 \\
\hline o + Double aortic arch repair & 1 \\
\hline - Single-patch technique. & 17 \\
\hline o Prior PAB & 1 \\
\hline PMVSD: & $58(35 \%)$ \\
\hline - Isolated & 30 \\
\hline$-+A^{5} D^{5} \|$ & 20 \\
\hline - + ASD II + TV6 repair & 1 \\
\hline - Prior PAB & 3 \\
\hline - + CoA repair & 2 \\
\hline$-+\mathrm{RPA}^{7}$ augmentation & 1 \\
\hline 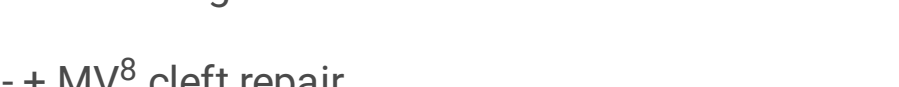 & 1 \\
\hline - + MV0 clett repair & $16(9.6 \%)$ \\
\hline TOF: & 11 \\
\hline - Isolated & 5 \\
\hline - Prior BTS ${ }^{9}$ & 3 \\
\hline - AVSD & 2 \\
\hline$-\mathrm{TOF}^{10} / \mathrm{PA}^{11}$ & $7(4.2 \%)$ \\
\hline Partial AVSD & $2(1.2 \%)$ \\
\hline Transitional AVSD & $6(3.6 \%)$ \\
\hline $\mathrm{PDA}^{12}$ ligation & $2(1.2 \%)$ \\
\hline CoA repair & $4(2.4 \%)$ \\
\hline Unbalanced AVSD (SV ${ }^{13} \rightarrow$ PAB/Glenn/Fontan) & $1(0.6 \%)$ \\
\hline Secundum ASD & \\
\hline
\end{tabular}




\begin{tabular}{|l|}
\hline Surgical procedures \\
\hline${ }^{1}$ Number \\
\hline${ }^{2}$ Complete Atrioventricular Septal Defect \\
\hline${ }^{3}$ Pulmonary Artery Band \\
\hline${ }^{4}$ Coarctation \\
\hline${ }^{5}$ Atrial Septal Defect \\
\hline${ }^{6}$ Tricuspid Valve \\
\hline${ }^{7}$ Right Pulmonary Artery \\
\hline${ }^{8}$ Mitral Valve \\
\hline${ }^{9}$ Blalock Taussig Shunt \\
\hline${ }^{10}$ Tetralogy of Fallot \\
\hline${ }^{11}$ Pulmonary Atresia \\
\hline${ }^{12}$ Patent Ductus Arteriosus \\
\hline${ }^{13}$ Single Ventricle \\
\hline
\end{tabular}


Table 5

Post-operative complications for the entire cohort.

\begin{tabular}{|ll|}
\hline Postoperative Complications (+ redo) & $\mathbf{N}^{1}$ \\
\hline Prolonged intubation & $25(12 \%)$ \\
Sepsis & $15(7.3 \%)$ \\
Wound infection & $5(2.5 \%)$ \\
Chylothorax & $10(4.9 \%)$ \\
\hline Persistent HB ${ }^{2}$ & $10(4.9 \%)$ \\
\hline Diaphragmatic palsy & $11(5.4 \%)$ \\
\hline JET & $4(2 \%)$ \\
\hline Pneumothorax & $5(2.5 \%)$ \\
\hline Neurological symptoms/insult & $6(2.9 \%)$ \\
\hline Post-operative bleeding & $9(4.4 \%)$ \\
\hline Vocal cord palsy & $2(1 \%)$ \\
\hline NEC & $3(1.5 \%)$ \\
\hline Open chest & $1(0.5 \%)$ \\
\hline Tracheostomy & $2(1 \%)$ \\
\hline${ }^{1}$ Number & $2(1 \%)$ \\
\hline${ }^{2}$ Heart Block & \\
\hline${ }^{3}$ Junctional Ectopic Tachycardia & \\
\hline${ }^{4}$ Necrotizing Enterocolitis & \\
\hline
\end{tabular}


Table 6

Pacemaker versus non-pacemaker groups comparison.

\begin{tabular}{|llll|}
\hline & Pacemaker & No Pacemaker & P-value \\
\hline $\mathrm{GA}^{1}$ & 36 & 37.3 & 0.3 \\
\hline Age $\left(\mathrm{m}^{2}\right)$ & 5.1 & 9 & 0.01 \\
\hline Weight $\left(\mathrm{kg}^{3}\right)$ & 3.9 & 5.6 & $<0.01$ \\
\hline $\mathrm{CPBT}^{4}\left(\right.$ mins $\left.^{5}\right)$ & 88.6 & 71.9 & 0.2 \\
\hline $\mathrm{ACCT}^{6}$ (mins) & 64.7 & 51.9 & 0.1 \\
\hline${ }^{1} \mathrm{Gestational} \mathrm{Age}$ & & \\
\hline${ }^{2}$ Months & & \\
\hline${ }^{3}$ Kilograms & \\
\hline${ }^{4}$ Cardiopulmonary Bypass Time & \\
\hline${ }^{5}$ Minutes & \\
\hline${ }^{6}$ Aortic Cross-Clamp Time \\
\hline
\end{tabular}


Table 7

Redo surgeries for the entire cohort.

\section{Re-do surgeries}

Complete AVSD2

- Two-patch technique:

o VSD ${ }^{3}$ closure

o $\mathrm{MV}^{4}+\mathrm{LVOTO}^{5}$ repair

o MV repair

o $\mathrm{MV}$ repair $\rightarrow$ replacement

- Single-patch technique.

o VSD/MV cleft repair

o VSD/MV/TV6 repair

o MV repair

Partial AVSD:

- MV repair

\section{PMVSD:}

- $\mathrm{CoA}^{7}$ repair $\rightarrow \mathrm{AAA}^{8}$ repair

AVSD/TOF:

- BTS $^{9} \rightarrow$ Stent BTS $\rightarrow$ Total repair with TAP ${ }^{10} \rightarrow$ Balloon dilation RPA ${ }^{11} \rightarrow$ RV-PA $^{12}$ homograft 1

+ residual VSD closure

- Total repair (valve-sparing) $\rightarrow$ MV repair

TOF/PA:

- BTS $\rightarrow$ VSD/RV-PA conduit $\rightarrow$ conduit replacement + LPA $^{13}$ plasty

${ }^{1}$ Number

${ }^{2}$ Atrioventricular Septal Defect

${ }^{3}$ Ventricular Septal Defect

${ }^{4}$ Mitral Valve

${ }^{5}$ Left Ventricular Outflow Tract Obstruction 
${ }^{6}$ Tricuspid Valve

${ }^{7}$ Coarctation

${ }^{8}$ Aortic Arch Aneurysm

${ }^{9}$ Blalock-Taussig Shunt

10 Transannular Patch

${ }^{11}$ Right Pulmonary Artery

${ }^{12}$ Right Ventricular - Pulmonary Artery

${ }^{13}$ Left Pulmonary Artery 
Table 8

Catheter interventions rates for the entire cohort.

\section{Catheter interventions}

Complete AVSD ${ }^{2}$ :

- Two-Parch technique (TPT)

o Balloon dilation of $\mathrm{CoA}^{3}$

o Para-valvar leak closure

COA:

- Balloon dilation

SV4:

- Sano shunt stent

- Stenting of MPA ${ }^{5}$ \& LPA ${ }^{6}$

AVSD/TOF7:

- BTS $^{8} \rightarrow$ Stent BTS $\rightarrow$ TAP $^{9} \rightarrow$ Balloon dilation RPA ${ }^{10} \rightarrow$ RV-PA $^{11}$ homograft + residual VSD ${ }^{12}$ closure

${ }^{1}$ Number

${ }^{2}$ Atrioventricular Septal Defect

${ }^{3}$ Coarctation

${ }^{4}$ Single Ventricle

${ }^{5}$ Main Pulmonary Artery

${ }^{6}$ Left Pulmonary Artery

${ }^{7}$ Tetralogy of Fallot

${ }^{8}$ Blalock-Taussig Shunt

${ }^{9}$ Transannular Patch

${ }^{10}$ Right Pulmonary Artery

${ }^{11}$ Right Ventricular - Pulmonary Artery

12 Ventricular Septal Defect 